\title{
An equation of the width of the depletion layer for a step heterojunction
}

\author{
M. Muhibbullah ${ }^{\mathrm{a}}$ M. Golam Mowla Choudhury ${ }^{\mathrm{b}}$ and Sharif M Mominuzzaman ${ }^{\mathrm{c}}$ \\ ${ }^{a}$ Department of Engineering Physics, Electronics and Mechanics \\ Nagoya Institute of Technology, Gokiso, Showa, Nagoya 466-8555, Japan \\ Tel-phone and Fax: 81052735 5581, e-mail: mmuhibbullah@yahoo.com \\ ${ }^{\mathrm{b}}$ Department of Electronics and Telecommunication Engineering, \\ Daffodil International University, 102 Shukrabad, Dhanmondi, \\ Dhaka-1207, Bangladesh \\ Tel-phone: 88029116774 Ex-133, e-mail: gmcw9f@daffodilvarsity.edu.bd \\ 'Department of Electrical and Electronic Engineering, \\ Bangladesh University of Engineering and Technology (BUET), \\ Dhaka-1000, Bangladesh \\ Phone: +88-02-8611594; +8802-9665650 80/Ext. 7770 (Off), 7113 (Dept) \\ Fax: +88-02-8611594, e-mail: momin@eee.buet.ac.bd.
}

For solar cell application open circuit voltage and short circuit current influence the solar efficiency directly. Carrier concentration, width of the depletion layer and other some related parameters influence on the amount of the open circuit voltage and short circuit current. Some equations can give the idea to improving the solar cell efficiency of a homo or heterojunction. For this a relation among the width of the depletion layer, carrier concentration and static electric potential of a step heterojunction has derived by the help of Gauss law.

Key words: Heterojunction, solar cell, step pn-junction, depletion layer

\section{INTRODUCTION}

To improve the solar cell efficiency it is necessary to increase both the open circuit voltage and short circuit current of a solar cell. An electric field in the depletion region of a cell acts as a force to separate the carriers. The carrier separation rate is directly proportional to the intensity of this electric field. The electric field is produced in the junction region by exchanging the charge carrier (such as electron) from one side (n-type region) to other side (p-type region) without any external biasing. The amount of exchanging electron and hole in the pn junction depends on the amount of the difference of Fermi energy level to their materials. Difference of Fermi energy gradually decreases by the carrier exchanging in the junction. Carrier exchanging is stopped when difference of Fermi energy levels become zero in the both p-type and n-type sides, and then the static electric field become maximum. In this way contact area and near the contact area become deplete of the carriers. The electric field of the depletion region produces a potential. This potential barrier, $\mathrm{V}_{\mathrm{b}}$, is not measurable by the external voltage meter. In the forward bias, a minimum bias voltage (equal and opposite of $\mathrm{V}_{\mathrm{b}}$ ) called Threshold voltage is needed to flow current to the junction. So, $V_{b}=-V_{t h}$, and Threshold voltage (as well as $\mathrm{V}_{\mathrm{b}}$ ) is measurable by help of the I-V characteristic measurement. Carrier generation of the solar cell depends on photosensitivity of their materials, and carrier separation depends on the static potential $\mathrm{V}_{\mathrm{b}}$. So, the open circuit voltage $\left(\mathrm{V}_{\mathrm{op}}\right)$ of a solar cell cannot exceed the static potential (i.e. $V_{\text {op }} \leq$ $\mathrm{V}_{\mathrm{b}}$ ), because the open circuit voltage is produced by the static potential. To gather better knowledge about open circuit voltage and short circuit current of a junction, it is important to understand the static potential $\mathrm{V}_{\mathrm{b}}$ and its related electric field. The width of the depletion layer gives us the idea about the electric field (Equations 20 \& 23) for the heterojunction. There is a popular equation [1] of width of the depletion layer for step heterojunction with external applied voltage. Also, many authors established an equation of step homo junction [1-3]. Our equations are the same as their equations. Difference is that those equations are derived from Poisson's equation [1-3]. Our equation is derived from the Gauss equation and from the different angle of our own ideas, and the derivation of this way is the first time work. Many researchers are doing the research work on the heterojunction solar cell. So, it may useful for the heterojunction solar cell applications.

\section{THEORY}

A semiconductor chip of wide band gap is used as a window layer and other semiconductor chip of narrow band gap is used as an absorption layer to the heterojunction solar cell. Usually wide band gap of binary compound is n-type so n-type thin layer is used as a window layer in the heterojunction solar cell. Let us consider the effective carrier concentration of n-type 
(window) chip $=N_{d}-N_{a}{ }^{\prime}=n_{n}\left(\mathrm{~cm}^{-3}\right)$

and, the effective carrier concentration of p-type (absorption) chip $=N_{a}-N_{d}{ }^{\prime}=n_{p}\left(\mathrm{~cm}^{-3}\right)$

Here $N_{d}$ and $N_{a}{ }^{\prime}$ are donor and acceptor concentration of n-type chip respectively, and $N_{a}$ and $N_{d}$ 'are acceptor and donor concentration of p-type chip respectively. In the monolithic deposition technique there are many minority carriers in the second and third layer of a device but in the deposition techniques of heterojunction, possibility of minority carrier is less. To deposit heterojunction second layer is deposited just on the first layer. So, there is no common part of the n-type and p-type layers to the heterojunction. Therefore the junction is step junction. We consider $N_{a}$ ' $\approx N_{d}{ }^{\prime} \approx 0$. In practical case, there are many defects in a semiconductor. To avoid the complicacy we can consider the carriers (donors and acceptors) are arranged homogeneously in the semiconductor without others defects.

Let us consider an ideal "cube" in which only one carrier is remaining in the center of this saying cube. These cubes build the whole chip, and $n_{n}$ and $n_{p}$ indicate the numbers of the cube per unit volume to n-type and p-type chip respectively.

So, volume of a cube (not actual volume of a carrier)

$$
\text { of n-type chip, } v_{n}=\frac{1}{n_{n}}\left(\mathrm{~cm}^{3}\right) \text {.... }
$$

And, volume of a cube of p-type chip,

$$
v_{p}=\frac{1}{n_{p}}\left(\mathrm{~cm}^{3}\right)
$$

Then, the length of an edge of the cube is;

$$
\begin{aligned}
& \text { for n-type chip } l_{n}=\frac{1}{n_{n}^{\frac{1}{3}}}(\mathrm{~cm}) . \\
& \text { for p-type chip } l_{p}=\frac{1}{n_{p}^{\frac{1}{3}}}(\mathrm{~cm})
\end{aligned}
$$

Area of one surface of the cube is;

$$
\begin{aligned}
& \text { for n-type chip }=\frac{1}{n_{n}^{\frac{2}{3}}}\left(\mathrm{~cm}^{2}\right) . \\
& \text { for p-type chip }=\frac{1}{n_{p}^{\frac{2}{3}}}\left(\mathrm{~cm}^{2}\right)
\end{aligned}
$$

We consider a very thin (thickness is $l_{p}$ in the p-type region) plane in parallel to the contact surface with a single cube across the thickness. The planes in parallel cover the chip.

If the total area of the cross section of the chip is ' $A$ ' $\mathrm{cm}^{2}$ then the number of the involve carriers (cube) of the one plane are;

$$
\begin{aligned}
& \text { for n-type side }=A n_{n}^{\frac{2}{3}} \\
& \text { for p-type side }=A n_{p}^{\frac{2}{3}}
\end{aligned}
$$

In intimate contact of two chips, some electrons will flow for difference of Fermi energy levels from n-type region to p-type region through the contact area (Fig. $1)$. By this way makes an electric field consequent electric potential in the contact region, which acts as an opposite force of the 'cause' of electron flow. This electron transport would continue until Fermi energy levels become equal in both sides. Then, there are no carriers in or near the contact area to both n-type and p-type sides called depletion region (Fig. 1). It is clear that this electron transport (to build $V_{b}$ ) depends only on active carrier concentration $\left(N_{d}-N_{a}{ }^{\prime}=n_{n}\right)$, and does not depend on the absolute carrier concentration of both sides. Here we have considered $N_{a}{ }^{\prime} \approx N_{d}{ }^{\prime} \approx 0$ thus, $N_{d}=n_{n}$ and $N_{a}=n_{p}$. So, we can consider donor and acceptor concentration by $\mathrm{n}_{\mathrm{n}}$ and $\mathrm{n}_{\mathrm{p}}$ instead to $\mathrm{N}_{\mathrm{d}}$ and $\mathrm{N}_{\mathrm{a}}$ in the depletion region although there is no carrier in the depletion region. In the equilibrium (equal

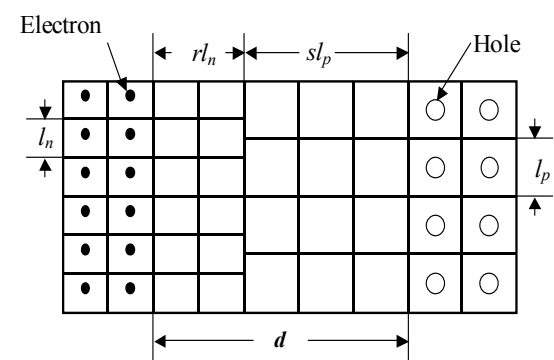

Fig. 1: Schematic diagram of a step heterojunction. Solid circles $(\bullet)$ represent electrons and open circles (o) illustrate holes.

Fermi energies to both sides) the total numbers of engage carriers (active donors and acceptors) in depletion region are;

$r A \boldsymbol{n}_{n}^{\frac{2}{3}}={ }_{s A} \boldsymbol{n}_{p}^{\frac{2}{3}}$

Here, $r$ and $s$ are integers, which indicate the plane number of n-type side and p-type side in the depletion region respectively. As for example $\mathrm{r}=2$ and $\mathrm{s}=3$ according the Fig. 1.

$$
\therefore s=\frac{r n_{n}^{\frac{2}{3}}}{n_{p}^{\frac{2}{3}}}
$$

So, the charges of the each side of the contact area are; $q=r A \boldsymbol{n}_{n}^{\frac{2}{3}} e=s A \boldsymbol{n}_{p}^{\frac{2}{3}} e$ (Coulomb).

And the width of depletion layer is;

$$
d=r l_{n}+s l_{p}=\frac{r}{n_{n}^{\frac{1}{3}}}+\frac{s}{n_{p}^{\frac{1}{3}}}(\mathrm{~cm}) \ldots .(11)
$$

Using the equation (3), (4) and (9) 


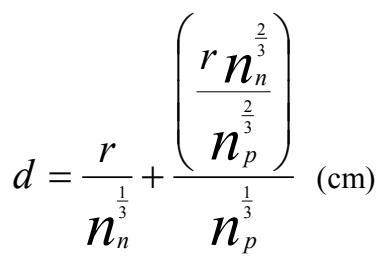

or, $r^{2}=\frac{d^{2} n_{n}^{\frac{2}{3}} n_{p}^{2}}{\left(n_{p}+n_{n}\right)^{2}}$

The Gauss law of static electric field, $\vec{E}$, is;

$K \in{ }_{o} \oint \vec{E} \cdot d \vec{S}=q$ (Coulomb)...

$K$ is the relative permittivity of the substance and $\mathrm{q}$ is the total electric charge of inside of the close Gauss surface $\oint d \vec{S}$.

Let us consider a close Gauss surface (Fig. 2) in the depletion region of n-type layer. There is no electric flux flow through the five Gauss surfaces which are in outside of the depletion region and the electric flux flow only through the Gauss surface of inside of the depletion region because that surface is staying in between the equal and opposite charges, and direction of electric field is the same as the direction of

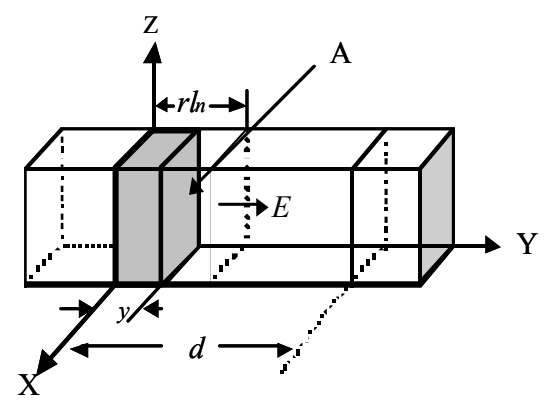

Fig. 2: Close Gauss surface to measure the electric field intensity in the depletion region of a step heterojunction.

the Gauss surface. Here the surface in the depletion region is situated on the distance ' $y$ ' from the origin. The value of electric field $E$ with $\oint d S=A \mathrm{~cm}^{2}$ is

$K_{n} \in{ }_{o} E A=q=e n_{n} y A$

$K_{n}$ is relative permittivity for n-type semiconductors. If we consider an others Gauss surface at distance ' $y+d y$ ' from the origin then the electric field $E+d E$ is as

$K_{n} \in_{o}(E+d E) A=q^{\prime}=e n_{n}(y+d y) A$

So, $K_{n} \in_{o} d E=e n_{n} d y$

By integration of above equation

$K_{n} \in{ }_{o} E=e n_{n} y+C$
When $\mathrm{y}=0$, then $E=0$, and when $\mathrm{y}=r l_{n}$, then $E=$ $E_{\mathrm{m}}$ (maximum), so, $C=0$

and $K_{n} \in{ }_{o} E=e n_{n} y$

and $K_{n} \in_{o} E_{m}=e n_{n} r l_{n}$

The equation (16) is linier so, we can draw the line of the electric field as Fig. 3(a).

Similarly, for p-type layer we can write,

$K_{p} \in{ }_{o} E=-e n_{p} y+K_{p} \in{ }_{o} E_{m}$

and, $K_{p} \in{ }_{o} E_{m}=e n_{p} s l_{p}$

$K_{p}$ is relative permittivity for p-type semiconductors. Minus sign indicates electric field intensity decreases with distance (y). Here, when y $=0$, then $E=E_{\mathrm{m}}$ (maximum), and when $\mathrm{y}=r l_{n}$, then $E=0$ so, $C=$ $K_{p} \in{ }_{o} E_{m}$.

Considering both n-type and p-type chips we can draw the electric field in depletion region as Fig. 3(b). From Fig. 3(b) the relation between electric field and potential difference $V_{b}$ is written (with avoiding the minus sign) as;

$V_{b}=\frac{E_{m}}{2}\left(r l_{n}+s l_{p}\right)=\frac{E_{m}}{2} d$

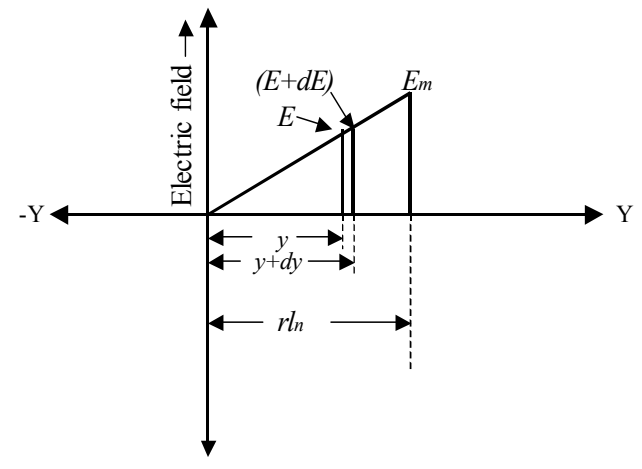

(a)

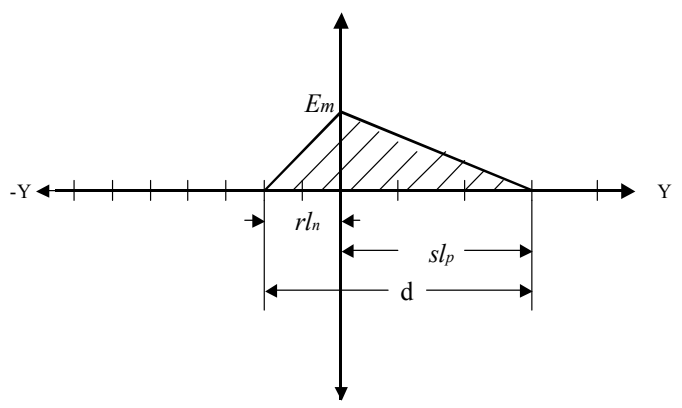

(b)

Fig. 3: Electric field in the depletion region of a step heterojunction.

$V_{b}$ is the static potential barrier across the width of the depletion layer.

Using equation (17), (19) and (20) we get; 


$$
V_{b}=\frac{e n_{n} r^{2} l_{n}{ }^{2}}{2 K_{n} \in_{o}}+\frac{e n_{p} s^{2} l_{p}{ }^{2}}{2 K_{p} \in_{o}}
$$

Putting the values of $l_{n}, l_{p}$, and $s$ from the equations (3), (4) and (9) into the equation (21) we get

$$
r^{2}=\frac{2 V_{b} \in_{o} K_{n} K_{p} n_{p}}{n_{n}^{\frac{1}{3}} e\left(K_{p} n_{p}+K_{n} n_{n}\right)}
$$

From equation (12) and (22) we get,

$$
\begin{aligned}
& \frac{d^{2} n_{n}^{\frac{2}{3}} n_{p}^{2}}{\left(n_{p}+n_{n}\right)^{2}}=\frac{2 V_{b} \in_{o} K_{n} K_{p} n_{p}}{n_{n}^{\frac{1}{3}} e\left(K_{p} n_{p}+K_{n} n_{n}\right)} \\
& \therefore d=\left(n_{p}+n_{n}\right) \sqrt{\frac{2 V_{b} \in_{o} K_{n} K_{p}}{e n_{p} n_{n}\left(K_{p} n_{p}+K_{n} n_{n}\right)} \cdots}
\end{aligned}
$$

We can calculate the width of the depletion layer d using the known value of $V_{b}, n_{n}$ and $n_{p}$ by the above equation (23) [1]. For homo junction as for example p-type and n-type $\mathrm{CuO}$ then $K_{p}=K_{n}=K$ (say), then

$$
d=\sqrt{\frac{2 V_{b} \in_{o} K\left(n_{p}+n_{n}\right)}{n_{p} n_{n} e}}
$$

This is the known equation [1-3] for step homo junction.

If $n_{n} \approx n_{p}=n$ (say) then,

$d=\sqrt{\frac{4 V_{b} \in_{0} K}{n e}}$

\section{CONCLUSIONS}

The relation among the width of the depletion layer, carrier concentration and static electric potential of a step heterojunction has derived by the help of Gauss law.

\section{ACKNOWLEDGMENT}

The authors are grateful to Professor Dr. Masaya Ichimura for his useful discussions.

\section{REFERENCES}

[1].Bart Van Zeghbroeck, Principles of Semiconductor Devices, Chapter 4, eBook, 2011.

[2]. S. M. Sze and Kwok K. Ng, Physics of Semiconductor Devices, WILEY-INTERSCIENCE, New Jersey, USA, 2007, $3^{\text {rd }}$ Edition.

[3]. A. S. Grove, Physics and Technology of Semiconductor Devices, Berkeley, New York: Wiley, 1967.

(Received 31 December 2011; Accepted 6 August 2012) 